\title{
NEURONAL CEROID LIPOFUSCINOSES
}

\author{
A CLINICAL AND MORPHOLOGICAL STUDY OF \\ 17 PATIENTS FROM SOUTHERN BRAZIL
}

\author{
ANA CRISTINA S PUGA****, LAURA B JARDIM*, LEILA CHIMELLI**, \\ CAROLINA FM DE SOUZA*, MARTA CLIVATI**
}

\begin{abstract}
The neuronal ceroid lipofuscinoses (NCL) are a group of inherited progressive neurodegenerative disorders with presentation from infancy to adulthood. Three main childhood forms can be established on the basis of age of onset, clinical course, and ultrastructural morphology: infantile (INCL), late infantile (LINCL), and juvenile (JNCL). Several variant subtypes have been described. Genetic and biochemical analysis are helping to better understand, diagnose and classify these disorders. We report on clinical, neurophysiological, neuroradiological, and morphological data from 17 patients with different forms (infantile, late infantile, and juvenile ) of neuronal ceroid lipofuscinoses (NCL) evaluated at Hospital de Clínicas de Porto Alegre, Southern Brazil, during 6 years (1992-1997). Seven cases were infantile; 5 were late infantile; and 5 were juvenile NCL. Gender ratio was male:female, 11:6. Age at presentation varied from 2-24 months for INCL; 2,5 to 5 years for LINCL ; and 4-10 years for the JNCL cases. Seizures (6 patients) and psychomotor retardation (1 patient) were the initial symptoms in the INCL group. All the patients in the group of LINCL had the usual findings. JNCL patients manifested different initial symptoms, although tending to follow a similar clinical picture within familial cases. Epidemiological data on the prevalence of NCLs in Brazil are not available, we expect this series of cases to contribute to further research in our population.
\end{abstract}

KEY WORDS: neuronal ceroid lipofuscinoses, lysosomal storage disease, Haltia-Santavuori disease, Jansky-Bielschowsky disease, Batten-Spielmeyer-Vogt disease.

\section{Lipofuscinoses ceróides neuronais: estudo clínico e morfológico de 17 pacientes do Sul do Brasil}

RESUMO - As lipofuscinoses ceroides neuronais (LCN) constituem um grupo de desordens neurodegenerativas, progressivas e de origem genética. O início dos sintomas varia desde a infancia até a vida adulta. Três principais formas infantis são estabelecidas com base na idade de início, evolução clínica e morfologia celular, através de microscopia eletrônica: infantil (LCNI), infantil tardia (LCNIT) e juvenil (LCNJ). Vários subtipos têm sido descritos. Investigação genética e bioquímica vem possibilitando melhor entendimento, diagnóstico e classificação destas desordens. Relatamos achados clínicos, neurofisiológicos, neurorradiológicos e morfológicos de 17 pacientes com diferentes formas de LCN (infantil, infantil tardia e juvenil), avaliados no Hospital de Clínicas de Porto Alegre, durante 6 anos (1992-1997). Tivemos 7 diagnósticos de LCNI, 5 de LCNIT, e 5 casos de LCNJ. Proporção entre gêneros foi 11:6 (masculino:feminino). Convulsões (6 pacientes) e retardo psicomotor (1 paciente) foram os sintomas iniciais no grupo LCNI. Todos os casos de LCNIT tiveram achados usuais. Os pacientes com LCNJ apresentaram diferentes sintomas iniciais, embora tendendo a similaridade em casos familiais. Não há dados epidemiológicos sobre LCN no Brasil. Esperamos que esta serie de casos contribua para maior investigação deste grupo de desordens em nossa população.

PALAVRAS-CHAVE: lipofuscinoses ceróides neuronais, doença lisossômica de depósito, doença de Haltia-Santavuori, doença de Jansky-Bielschowsky, doença de Batten-Spielmeyer-Vogt.

*Serviço de Genética Médica, Hospital de Clínicas de Porto Alegre, Porto Alegre, Brazil; **Departamento de Patologia da Faculdade de Medicina de Ribeirão Preto da Universidade de São Paulo, Brazil. ***Division of Genetics, The Children's Hospital, Harvard Medical School, Boston, MA, USA, recipient of scholarship by CAPES (Fundação Coordenação de Aperfeicoamento de Pessoal de Nivel Superior), Brasilia, Brazil. Aceite: 11-maio-2000.

Dr. Ana Cristina Puga - Division of Genetics, The Children's Hospital - 300 Longwood Avenue, Fegan 10 Boston, MA, 02115, USA. Fax 1617277 5933.E-mail: puga@al.tch.harvard.edu 
The neuronal ceroid lipofuscinoses (NCLs) are a group of inherited, progressive neurodegenerative disorders in children and in adults characterized morphologically by two processes: accumulation of autofluorescent lipopigments within various cell types and loss of cells, especially in the cerebral cortex ${ }^{1-3}$. Clinical features consist of various combinations of seizures, psychomotor deterioration and blindness, the ages and order of onset differing for each $\mathrm{NCL}^{4}$. There is no cure available - treatment focus on management of symptoms - with patients progressing to a vegetative state and death ${ }^{2,4}$. The NCLs represent the most common group of autosomal recessive, progressive neurodegenerative disorders in childhood. Worldwide epidemiological data do not exist, but a higher incidence of NCL has been observed in northern countries ${ }^{5}$. The figure of 1.2: 100,000 life births has been quoted for all childhood forms ${ }^{6}$, but clinically different forms may occur in different frequencies in different regions ${ }^{4,5,7-10}$.

On light microscopy, all NCLs have lipopigments with staining characteristics similar to both ceroid, which occurs in conditions such as liver cirrhosis; and lipofuscin, a pigment which normally accumulates in old age ${ }^{3}$. Ultrastructurally, these lipopigments present as granular osmiophilic deposits (GRODs), curvilinear bodies (CB) and fingerprint profiles (FP), recognizable by electron microscopy (EM) especially in circulating lymphocytes and skin biopsies ${ }^{11}$.

Classically, the NCLs were divided in four major types, based on age at onset, clinical course, and ultrastructural morphology. They are: infantile NCL (INCL; Santavuori-Haltia disease; MIM256730) characterized ultrastructurally by GRODs; classical late-infantile NCL (LINCL; Jansky-Bielschowsky disease; MIM204500) characterized by curvilinear bodies; juvenile NCL (JNCL; Batten's disease or Spielmeyer-Vogt-Sjögren disease; MIM204200) characterized by fingerprint profiles; and adult NCL (ANCL; Kufs disease; MIM204300,162530) demonstrating FP, GRODs and CB $^{1,3,12-17}$. This simple and traditional classification has become blurred with the description of variants which overlap the major groups. The variants include a Finnish variant of LINCL (MIM256731); early-juvenile NCL and juvenile NCL with GRODs ${ }^{3,17-21}$. The atypical patients, over the last decade, have prompted the suggestion of 15 atypical variants and minor syndromes ${ }^{9,11}$. The clinical classification continues to be extremely useful, providing the first approach to this group of diseases and helping differential diagnosis to other neurodegenerative disorders. Nevertheless, with so many variants described, the limits are sometimes blurred and a more extensive classification of NCL was proposed ${ }^{22,23}$.

The identification of loci for infantile NCL and juvenile NCL on chromosomes 1 and 16 respectively, and the exclusion of linkage of late-infantile NCL to either one, in the early 1990's, prompted a reclassification of NCL disorders, based on molecular genetics: CLN1 for infantile NCL; CLN2 for classical late-infantile NCL; CLN3 for juvenile NCL and CLN4 for adult NCL ${ }^{18,24-29}$. To date, there are eight established forms of NCL (CLN 1-8), with CLN5, 6 and 7 being variants of late-infantile NCL. Four CLN genes have been isolated (CLN1, CLN2, CLN3 and CLN5), and based on these genes products, the neuronal ceroid lipofuscinoses are being more clearly recognized as lysosomal storage diseases (Table 1$)^{29}$.

The characterization of the NCLs as lysosomal storage diseases has been made possible by discovery of the lysosomal enzymes involved. In practice, these enzymes - palmitoyl-protein thioesterase for CLN1, pepinase for CLN2 and lysosomal membrane protein for CLN3 - should be routinely assayed for diagnostic purposes. However, diagnosis, in many countries, still depends on demonstration of ultrastructural patterns typical of these diseases.

We report a case series of 17 Brazilian patients affected by different types of NCL, concerning their clinical, neurophysiological, neuroradiological, and morphological findings.

\section{METHOD}

This series comprises patients referred to Hospital de Clinicas de Porto Alegre, Southern Brazil, for genetic investigation due to neurodegenerative disorders of unknown cause, between 1992 and 1997.

After exclusion of amino acidopathies, organic acidurias, adrenoleukodystrophy and other peroxisomal disorders and lysosomal storage disorders, patients were considered as candidates for ultrastructural analysis 
Table 1. The established human forms of neuronal ceroid-lipofuscinosis*.

\begin{tabular}{|c|c|c|c|c|c|c|c|c|}
\hline \multirow[b]{2}{*}{$\begin{array}{c}\text { Disease } \\
\text { entity }\end{array}$} & \multirow[b]{2}{*}{$\begin{array}{c}\text { Infantile } \\
\text { NCL } \\
\text { (INCL), } \\
\text { CLN1 }\end{array}$} & \multicolumn{4}{|c|}{ Late-infantile NCL (LINCL) } & \multirow[b]{2}{*}{$\begin{array}{c}\text { Juvenile } \\
\text { NCL } \\
\text { CLN3, } \\
\text { Batten }\end{array}$} & \multirow[b]{2}{*}{$\begin{array}{c}\text { No } \\
\text { epilepsy } \\
\text { CLN4 }\end{array}$} & \multirow[b]{2}{*}{ CLN8 } \\
\hline & & $\begin{array}{l}\text { CLN2, } \\
\text { "classical" }\end{array}$ & $\begin{array}{l}\text { CLN5, } \\
\text { Finnish } \\
\text { variant }\end{array}$ & $\begin{array}{l}\text { CLN6, } \\
\text { variant }\end{array}$ & $\begin{array}{l}\text { CLN7, } \\
\text { "Turkish" }\end{array}$ & & & \\
\hline $\begin{array}{l}\text { Age of } \\
\text { onset }\end{array}$ & $0-2 y$ & $2-4 y$ & $4-7 y$ & $4-5 y$ & $4-7 y$ & $4-9 y$ & $11-50 \mathrm{y}$ & $5-10 y$ \\
\hline $\begin{array}{c}\text { Morphology } \\
\text { EM } \\
\text { inclusions }\end{array}$ & GROD & $\mathrm{CB}$ & $\mathrm{CB} / \mathrm{FP}$ & $\mathrm{CB} / \mathrm{FP}$ & $\mathrm{CB} / \mathrm{FP}$ & FP & $\begin{array}{c}\text { FP; } \\
\text { GROD }\end{array}$ & $\begin{array}{c}\text { CB/ } \\
\text { GROD }\end{array}$ \\
\hline $\begin{array}{c}\text { Chromosomal } \\
\text { location }\end{array}$ & $\begin{array}{l}1 \mathrm{p} \\
32\end{array}$ & $\begin{array}{c}11 \mathrm{p} \\
15\end{array}$ & $\begin{array}{c}13 q \\
22\end{array}$ & $\begin{array}{c}15 q \\
21-23\end{array}$ & Unknown & $\begin{array}{c}16 \mathrm{p} \\
12\end{array}$ & Unknown & $\begin{array}{l}8 \mathrm{p} 22 \\
- \text { Ter }\end{array}$ \\
\hline $\begin{array}{c}\text { Gene } \\
\text { product }\end{array}$ & $\begin{array}{l}\text { Palmitoyl- } \\
\text { protein } \\
\text { thioesterase }\end{array}$ & Pepinase & $\begin{array}{c}\text { Membrane } \\
\text { protein }\end{array}$ & Unknown & Unknown & $\begin{array}{c}\text { Lysosomal } \\
\text { membrane } \\
\text { protein }\end{array}$ & UnknownL & Unknown \\
\hline
\end{tabular}

*Adapted from Bennet and Hofmann, 1999.

using skin biopsy. In this five-years-period, 3634 patients have been evaluated in our genetic outpatient clinics, 453 showing neurological deterioration as their main problem. Twenty five patients met the criteria for ultrastructural analysis, ages varying from 4 months to 45 years.

Patients were evaluated by clinic and neurologic examination, neurometabolic investigation, electrophysiological studies [ electroencephalogram (EEG) with photic stimulation and visual-evoked potential (VEP)], ophthalmological examination and neuroimaging [computed tomography (CT) and/or magnetic resonance imaging (MRI)]. VEP studies were available for 7 patients. Somatosensory-evoked potential (SEP) studies and electroretinogram (ERG) recordings were not available at this time. All cases were then submitted to skin biopsy. The tissue was immediately fixed in $2.5 \%$ glutaraldehyde in phosphate buffer. It was post-fixed in osmium tetroxide and processed for resin embedding. One-micron sections were stained with toluidine blue. Ultra-thin sections, from selected areas were stained with lead citrate and uranile acetate and examined in a Zeiss 109 electron microscope.

Cases were classified mainly on the basis of clinical manifestations, age of onset, EEG patterns and EM observations $^{5,11,18,30-32}$. The cases were grouped into INCL, 7 patients; LINCL, 5 patients; JNCL, 5 patients.

\section{RESULTS}

Detailed descriptions of the results are summarized in Tables 2, 3 and 4.

\section{Infantile neuronal ceroid lipofuscinosis - 7 cases (Table 2)}

Clinical findings. Three patients were sibs (Patients 3, 4 and 5), Patients 1 and 2 were isolated cases. Age of onset within the group ranged from 3-24 months. Seizures were the most frequent presenting symptom, shortly followed by psychomotor regression or delay, myoclonus and blindness. Ophthalmological examination showed macular degeneration with optic atrophy within 8-24 months of disease onset. Patients 6 and 7 were uncle and nephew, respectively (Fig 1). Patient 7 followed a clinical course typical of INCL. Patient 6, however, presented an atypical progression of disease. Despite the early-developing symptoms, rapid evolution to a difficult-totreat myoclonic epilepsy and blindness, this patient was alive at the age of 19 years, although in a vegetative state. A twin brother similarly affected, with the same age of onset, died at age 2 years of pulmonar infection without proper neurological or morphological investigation. All the other patients died between 2.5 and 6 years. 
Table 2. Infantile neuronal ceroid lipofuscinosis.

\begin{tabular}{|c|c|c|c|c|c|c|c|c|c|c|}
\hline Patient & $\begin{array}{l}\text { Family } \\
\text { History }\end{array}$ & $\begin{array}{l}\text { Sex } \\
(\mathrm{m}\end{array}$ & $\begin{array}{l}\text { Age } \\
\text { at } \\
\text { onset } \\
\text { nonths) }\end{array}$ & $\begin{array}{l}\text { Presenting } \\
\text { Symtoms }\end{array}$ & $\begin{array}{l}\text { Subsequent } \\
\text { symtoms } \\
\text { during first } \\
5 \text { years }\end{array}$ & $\begin{array}{c}\text { EEG } \\
\text { (months) }\end{array}$ & $\begin{array}{c}\text { VEP } \\
\text { (months) }\end{array}$ & $\begin{array}{l}\text { CT-MR } \\
\text { Atrophy } \\
\text { and age } \\
\text { (months) }\end{array}$ & $\begin{array}{c}\text { Electron } \\
\text { micros- } \\
\text { copy }\end{array}$ & $\begin{array}{c}\text { Retinal } \\
\text { findings } \\
\text { (months) }\end{array}$ \\
\hline 1 & NO & $\mathrm{M}$ & 3 & Seizures & $\begin{array}{c}\text { Psychomotor } \\
\text { retardation } \\
\text { Myoclonus } \\
\text { Visual } \\
\text { failure }\end{array}$ & $\begin{array}{c}\text { Severe } \\
\text { disorganized } \\
\text { background } \\
\text { activity } \\
\text { generalized } \\
\text { slow, SW (12) }\end{array}$ & $\begin{array}{r}\text { Low } \\
(12)\end{array}$ & $\begin{array}{c}\text { Cerebral } \\
\text { (12) }\end{array}$ & $\begin{array}{c}\text { GROD/ } \\
\text { FP }\end{array}$ & $\begin{array}{c}\text { Macular } \\
\text { degeneration } \\
\text { (15) }\end{array}$ \\
\hline 2 & NO & F & 15 & Seizures & $\begin{array}{l}\text { Psichomotor } \\
\text { retardation } \\
\text { Visual failure } \\
\text { Worsering } \\
\text { of seizures }\end{array}$ & $\begin{array}{l}\text { Generalized } \\
\text { diminution } \\
\text { of wave } \\
\text { amplitudes } \\
\text { (24) }\end{array}$ & $\begin{array}{l}\text { Absent } \\
(24)\end{array}$ & $\begin{array}{l}\text { Cerebral } \\
\text { and } \\
\text { cerebellar } \\
(24)\end{array}$ & $\begin{array}{c}\text { GROD/ } \\
\text { FP }\end{array}$ & $\begin{array}{c}\text { Macular } \\
\text { degeneration } \\
\text { (36) }\end{array}$ \\
\hline 3 & YES & $\mathrm{F}$ & 8 & Seizures & $\begin{array}{l}\text { Psychomotor } \\
\text { regression } \\
\text { Dementia } \\
\text { Spacticity } \\
\text { Visual failure }\end{array}$ & $\begin{array}{l}\text { Disorganized } \\
\text { background } \\
\text { activity, focal } \\
\text { paroxysm } \\
\text { (10) }\end{array}$ & - & $\begin{array}{c}\text { Cerebral } \\
\text { (18) }\end{array}$ & $\begin{array}{l}\mathrm{CB} / \\
\mathrm{FP}\end{array}$ & $\begin{array}{c}\text { Macular } \\
\text { degeneration } \\
\text { (24) }\end{array}$ \\
\hline 4 & YES & $\mathrm{F}$ & 2 & Seizures & $\begin{array}{l}\text { Psychomotor } \\
\text { retardation } \\
\text { Dementia } \\
\text { Myoclonus } \\
\text { Visual } \\
\text { failure a } \\
\text { Choreoathetosi }\end{array}$ & $\begin{array}{l}\text { Disorganized } \\
\text { activity and } \\
\text { generalized } \\
\text { diminution } \\
\quad \text { of wave } \\
\text { amplitudes (12) } \\
\text { is }\end{array}$ & $\begin{array}{l}\text { Absent } \\
\text { (12) }\end{array}$ & $\begin{array}{l}\text { Cerebral } \\
\text { (12) }\end{array}$ & $\begin{array}{l}\text { GROD/ } \\
\text { FP }\end{array}$ & $\begin{array}{c}\text { Macular } \\
\text { degeneration } \\
\text { (12) }\end{array}$ \\
\hline 5 & YES & M & 24 & Seizures & $\begin{array}{c}\text { Psychomotor } \\
\text { regression } \\
\text { Dementia }\end{array}$ & $\begin{array}{l}\text { Disorganized } \\
\text { background } \\
\text { activity, } \\
\text { diminution of } \\
\text { wave amplitudes } \\
\text { (24) }\end{array}$ & es & $\begin{array}{c}\text { Cerebral } \\
\text { (30) }\end{array}$ & $\begin{array}{c}\text { GROD/ } \\
\text { CB }\end{array}$ & $\begin{array}{c}\text { Macular } \\
\text { degeneration } \\
\text { (36) }\end{array}$ \\
\hline 6 & YES & M & $\begin{array}{ll}4 & \mathrm{P} \\
& \end{array}$ & $\begin{array}{l}\text { Psychomotor } \\
\text { retardation }\end{array}$ & $\begin{array}{c}\text { Seizures } \\
\text { Myoclonus } \\
\text { Dementia } \\
\text { Visual } \\
\text { failure }\end{array}$ & $\begin{array}{l}\text { Disorganized } \\
\text { background } \\
\text { activity, } \\
\text { flattening of } \\
\text { waves (30) }\end{array}$ & - & $\begin{array}{c}\text { Cerebral } \\
(48)\end{array}$ & $\begin{array}{l}\text { GROD/ } \\
\text { CB }\end{array}$ & - \\
\hline 7 & YES & M & 5 & Seizures & $\begin{array}{c}\text { Psychomotor } \\
\text { regression } \\
\text { Dementia }\end{array}$ & $\begin{array}{c}\text { Focal } \\
\text { paroxysm } \\
\text { disorganized } \\
\text { background } \\
\text { activity (5) }\end{array}$ & $\begin{array}{l}\text { Absent } \\
\text { (12) }\end{array}$ & $\begin{array}{c}\text { Cerebral } \\
\text { (12) }\end{array}$ & $\begin{array}{l}\mathrm{CB} / \\
\mathrm{FP}\end{array}$ & $\begin{array}{c}\text { Macular } \\
\text { degeneration } \\
\text { (18) }\end{array}$ \\
\hline
\end{tabular}

GROD osmiophilic bodies, FP fingerprint profiles, CB curvilinear bodies, SW spike and wave, - not performed.

Neurophysiological findings. The earliest EEG finding was a disorganized background activity. Paroxysms, either generalized or focal were recorded in patients 1,3, and 7. Slowing or flattening of wave amplitude was present within 1- 26 months of disease onset. VEP was low in patient 1, after 9 months of symptoms; and absent in patients 2, 4, and 7 within 7-19 months of evolution.

Neuroradiological findings. CT and/or MRI studies revealed varying degrees of cerebral and/or cerebellar atrophy in all patiens, within 3 years of onset of disease, most frequently within 8-10 months. 
Table 3. Late infantile neuronal ceroid lipofuscinosis.

\begin{tabular}{|c|c|c|c|c|c|c|c|c|c|}
\hline Patient & $\begin{array}{l}\text { Family } \\
\text { History }\end{array}$ & Sex & $\begin{array}{c}\text { Age at } \\
\text { onset } \\
\text { (years) }\end{array}$ & $\begin{array}{l}\text { Presenting } \\
\text { Symtoms }\end{array}$ & $\begin{array}{l}\text { Subsequent } \\
\text { symtoms } \\
\text { during } \\
\text { first } \\
5 \text { years }\end{array}$ & $\begin{array}{l}\text { EEG } \\
\text { (years) }\end{array}$ & $\begin{array}{l}\text { VEP } \\
\text { (years) }\end{array}$ & $\begin{array}{l}\text { CT- } \\
\text { MR } \\
\text { Atrophy } \\
\text { and age } \\
\text { (Years) }\end{array}$ & $\begin{array}{l}\text { Electron Retinal } \\
\text { micros- findings } \\
\text { copy }\end{array}$ \\
\hline 8 & YES & M & $4 \mathrm{~F}$ & $\begin{array}{c}\text { Psychomotor } \\
\text { retardation }\end{array}$ & $\begin{array}{c}\text { Seizures } \\
\text { Myoclonus } \\
\text { Irritability }\end{array}$ & $\begin{array}{c}\text { Severe } \\
\text { background } \\
\text { disorganization } \\
\text { Large } \\
\text { polyspikes } \\
\text { after photic } \\
\text { stimulation } \\
(4,5)\end{array}$ & - & $\begin{array}{l}\text { Normal } \\
\text { (4) }\end{array}$ & $\begin{array}{l}\text { CB/ Macular } \\
\text { FP degeneration }\end{array}$ \\
\hline 9 & NO & M & 2.5 & $\begin{array}{c}\text { Clumsiness } \\
\text { Seizures }\end{array}$ & $\begin{array}{l}\text { Myoclonic } \\
\text { epilepsy } \\
\text { Ataxia } \\
\text { Spasticity }\end{array}$ & $\begin{array}{c}\text { Large } \\
\text { SW } \\
\text { after photic } \\
\text { stimulation } \\
\quad(4)\end{array}$ & $\begin{array}{l}\text { Normal } \\
(2.5)\end{array}$ & $\begin{array}{c}\text { Cerebral } \\
\text { (3) }\end{array}$ & $\begin{array}{c}\mathrm{CB} / \\
\text { GROD }\end{array}$ \\
\hline 10 & YES & M & 4 & Hypotonia & $\begin{array}{l}\text { Psychomotor } \\
\text { regression } \\
\text { Visual } \\
\text { failure }\end{array}$ & $\begin{array}{l}\text { Background } \\
\text { disorganization } \\
+ \text { PS after } \\
\text { photic } \\
\text { stimulation (5) }\end{array}$ & ) & $\begin{array}{l}\text { Cerebral } \\
\text { and } \\
\text { Cerebellar } \\
(4.5)\end{array}$ & $\begin{array}{c}\text { GROD Macular } \\
\text { degeneration } \\
+ \text { pigmentar } \\
\text { aggregate }\end{array}$ \\
\hline 11 & YES & M & 5 & $\begin{array}{l}\text { Slurred } \\
\text { Speech }\end{array}$ & $\begin{array}{c}\text { Ataxia } \\
\text { Seizures } \\
\text { Myoclonus } \\
\text { Visual } \\
\text { failure } \\
\text { Dementia }\end{array}$ & $\begin{array}{c}\text { Severe } \\
\text { disorganized } \\
\text { background } \\
+ \text { PS after } \\
\text { photic } \\
\text { stimulation }\end{array}$ & $\begin{array}{l}\text { Normal } \\
\text { (5) }\end{array}$ & $\begin{array}{l}\text { Cerebral } \\
\text { cerebellar } \\
\text { (6) }\end{array}$ & $\begin{array}{c}\text { GROD/ Macular } \\
\text { CB degenration } \\
\text { + pigmentar } \\
\text { aggregate }\end{array}$ \\
\hline 12 & NO & M & $4 \mathrm{~F}$ & $\begin{array}{l}\text { Psychomotor } \\
\text { regression }\end{array}$ & $\begin{array}{c}\text { Ataxia } \\
\text { Seizures } \\
\text { Visual Failure } \\
\text { Dementia }\end{array}$ & $\begin{array}{l}\text { Desorga- } \\
\text { nized back- } \\
\text { ground }+\mathrm{PS} \\
\text { after photic } \\
\text { stimulation }(4)\end{array}$ & Normal & $\begin{array}{c}\text { Cerebellar } \\
\text { (4) }\end{array}$ & $\begin{array}{l}\text { CB/ Macular } \\
\text { FP degeneration }\end{array}$ \\
\hline
\end{tabular}

GROD osmiophilic bodies, FP fingerprint profiles, CB curvilinear bodies, SW spike and wave, - not performed, PS polyspikes.

Morphological findings. Ultrastructural patterns of deposits in skin biopsies showed different combinations of GRODs, curvilinear bodies (CB), and fingerprint profiles (FP); a range of findings beyond the traditionally expected pattern of osmiophilic bodies for INCL, but a diversity already described $^{28,33}$ (Figs 2 and 3).

\section{Late infantile neuronal ceroid-lipofuscinosis - 5 cases (Table 3)}

Clinical findings. Two patients were sibs (patients 10 and 11). Patient 8 had a brother who developed seizures and psychomotor regression by the age of 2.5 years, progressing with dementia and visual failure, and dying by the age of 9 years without a conclusive diagnosis. Age at onset in the group varied between $2.5-5$ years. Decline of mental capacities was the leading presenting symptom. Ataxia, seizures, dementia and visual failure followed in the majority (patients 10, 11, and 12). Ophthalmological examination revealed macular degeneration, associated to pigment aggregation in two cases ( patients 10 and 11). 


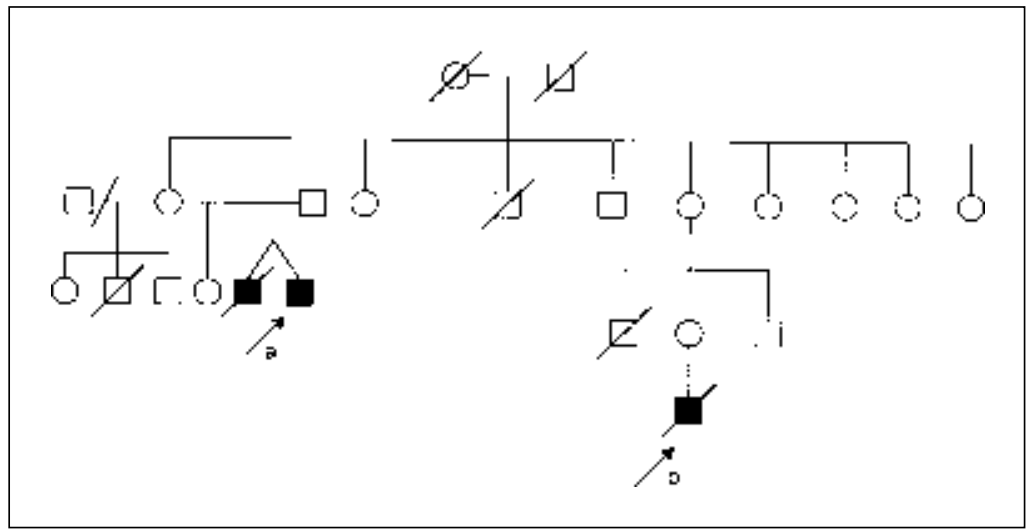

Fig 1. Heredogram for NCL1 family. (a) Patient 6; (b) Patient 7.

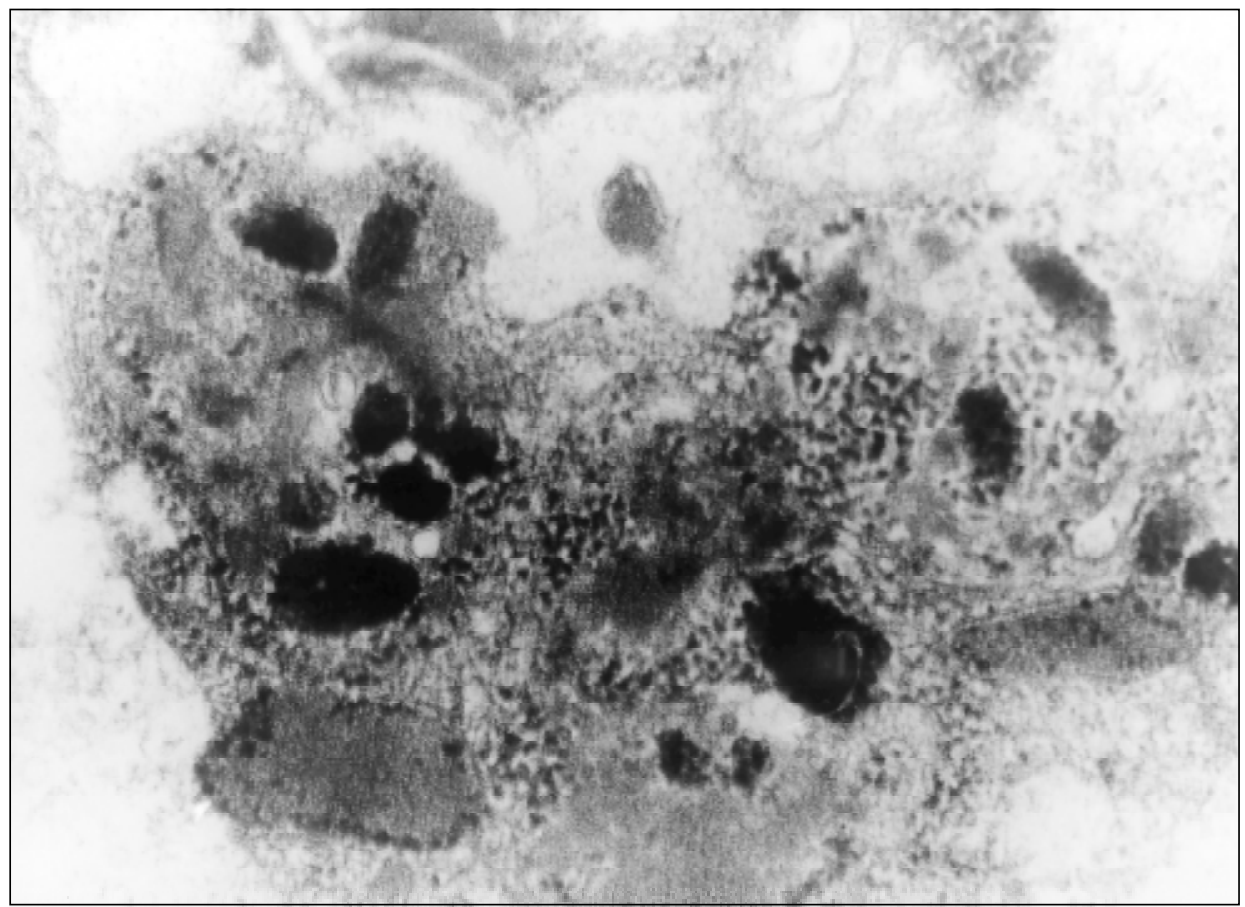

Fig 2. Osmiophilic and curvilinear bodies in macrophage, X 30,000.

Neurophysiological findings. The earliest EEG finding was a disorganized background activity. The specific feature of posterior spikes in response to low-frequency photic stimulation could be detected in all patients at ages 4-6 years. VEP was recorded in three cases (patients 9, 11, and 12), all of them normal, at $2.5,5$, and 4 years of age, respectively.

Neuroradiological findings. CT scan and/or MRI were performed within one year of disease onset, and showed different degrees of cerebral and cerebellar atrophy in all but patient 8 . 
Table 4. Juvenile neuronal ceroid lipofuscinosis

\begin{tabular}{|c|c|c|c|c|c|c|c|c|c|c|}
\hline Patient & $\begin{array}{l}\text { Family } \\
\text { History }\end{array}$ & Sex & $\begin{array}{c}\text { Age at } \\
\text { onset } \\
\text { (years) }\end{array}$ & $\begin{array}{l}\text { Presen- } \\
\text { ting } \\
\text { Symtoms }\end{array}$ & $\begin{array}{c}\text { Subsequent } \\
\text { symtoms } \\
\text { during first } \\
5 \text { years }\end{array}$ & $\begin{array}{l}\text { EEG } \\
\text { (years) }\end{array}$ & $\begin{array}{l}\text { VEP } \\
\text { (years) }\end{array}$ & $\begin{array}{c}\text { CT-MR } \\
\text { Atrophy } \\
\text { and age } \\
\text { (years) }\end{array}$ & $\begin{array}{l}\text { Electron } \\
\text { microscopy }\end{array}$ & $\begin{array}{l}\text { Retinal } \\
\text { findings }\end{array}$ \\
\hline 13 & YES & $\mathrm{M}$ & 9 & $\begin{array}{c}\text { Behavior } \\
\text { problems } \\
\text { and } \\
\text { isolationism }\end{array}$ & $\begin{array}{c}\text { Dementia } \\
\text { Visual } \\
\text { failure } \\
\text { Spasticity }\end{array}$ & $\begin{array}{l}\text { Background } \\
\text { disorgani- } \\
\text { zation }+ \\
\text { diffuse bursts } \\
\text { of slow } \\
\text { waves and } \\
\text { spikes (15) }\end{array}$ & $\begin{array}{l}- \\
- \\
-\end{array}$ & $\begin{array}{l}\text { Hypodensity } \\
\text { of white } \\
\text { matter } \\
\text { mainly on } \\
\text { temporo- } \\
\text { occipital } \\
\text { region } \\
\text { (12) }\end{array}$ & $\begin{array}{l}\text { GROD/ } \\
\text { FP d }\end{array}$ & $\begin{array}{l}\text { Macular } \\
\text { degeneration }\end{array}$ \\
\hline 14 & YES & F & 10 & $\begin{array}{l}\text { Psychotic } \\
\text { and } \\
\text { Aggressive } \\
\text { behavior }\end{array}$ & $\begin{array}{l}\text { Dementia } \\
\text { Seizures }\end{array}$ & $\begin{array}{l}\text { Background } \\
\text { disorgani- } \\
\text { zation }+ \\
\text { diffuse bursts } \\
\text { of slow } \\
\text { waves and } \\
\text { spikes (15) }\end{array}$ & - & $\begin{array}{l}\text { Hypodensity } \\
\text { of white } \\
\text { matter mainly } \\
\text { on temporo } \\
\text {-occipital } \\
\text { region } \\
\text { (15) }\end{array}$ & 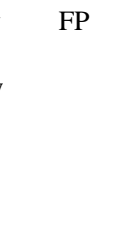 & Normal \\
\hline 15 & YES & $\mathrm{F}$ & $\begin{array}{ll}4 & \mathrm{P} \\
\end{array}$ & $\begin{array}{l}\text { Psychomotor } \\
\text { Regression }\end{array}$ & $\begin{array}{c}\text { Ataxia } \\
\text { Seizures } \\
\text { dementia } \\
\text { Visual } \\
\text { failure }\end{array}$ & $\begin{array}{c}\text { Background } \\
\text { disorgani- } \\
\text { zation }+ \\
\text { partial } \\
\text { paroxysm } \\
(10)\end{array}$ & - & $\begin{array}{l}\text { Cerebral } \\
\text { and } \\
\text { Cerebellar } \\
\text { (9) }\end{array}$ & $\begin{array}{c}\text { FP/ } \\
\text { GROD }\end{array}$ & Normal \\
\hline 16 & YES & $\mathrm{F}$ & 8 & Seizures & $\begin{array}{c}\text { Visual } \\
\text { failure } \\
\text { Dementia }\end{array}$ & $\begin{array}{c}\text { Severe } \\
\text { multifocal } \\
\text { paroxysm } \\
\text { (9) }\end{array}$ & - & $\begin{array}{c}\text { Cerebral } \\
\text { (9) }\end{array}$ & $\begin{array}{c}\text { FP/ } \\
\text { GROD }\end{array}$ & $\begin{array}{l}\text { Macular } \\
\text { degene- } \\
\text { ration }\end{array}$ \\
\hline 17 & YES & M & $5 \quad \mathrm{P}$ & $\begin{array}{l}\text { Psychomotor } \\
\text { regression }\end{array}$ & $\begin{array}{c}\text { Seizures } \\
\text { Visual } \\
\text { failure } \\
\text { Dementia }\end{array}$ & $\begin{array}{c}\text { Disorganized } \\
\text { background } \\
+ \\
\text { paroxysms }\end{array}$ & - & $\begin{array}{c}\text { Cerebral } \\
\text { (10) }\end{array}$ & FP & - \\
\hline
\end{tabular}

GROD osmiophilic bodies, FP fingerprint profiles, CB curvilinear bodies, SW spike and wave, - not performed

Morphological findings. Ultrastructural analysis of skin biopsies showed combinations of CB either with FP or with GRODs. In patient 10, only GRODs were found; but his brother's biopsy (patient 11) showed both GRODs and CB.

\section{Juvenile neuronal ceroid-lipofuscinosis - 5 cases (Table 4)}

Clinical findings. Here we had two sets of sibs (patients 13 and 14; patients 15,16, and 17). Age at onset ranged between 4-10 years. Behavioral problems were the initial symptoms in patients 13 and 14 , leading to dementia in 4-5 years. Psycomotor regression was the presenting symptom in patients 15 and 17; their sister (patient 16, with a later age of onset), presented with seizures. Patients 15 and 17 were blind by the age of 8 years. At 9 years, patients 16 was beginning to show visual failure. Ophthalmological examinations on patient 13 showed macular degeneration by the age 12 years. Patient 14 had a normal exam at 12 years old. Patients 13 and 14 were the only ones with available information on vacuolated lymphocytes; both exams were positive. Patients 13 and 15 died from complications of a severe myoclonic epilepsia by the ages of 17 and 11years, respectively. When last evaluated, patient 14 was 20 years old, presented a severe epileptic disorder and was in a vegetative state. 


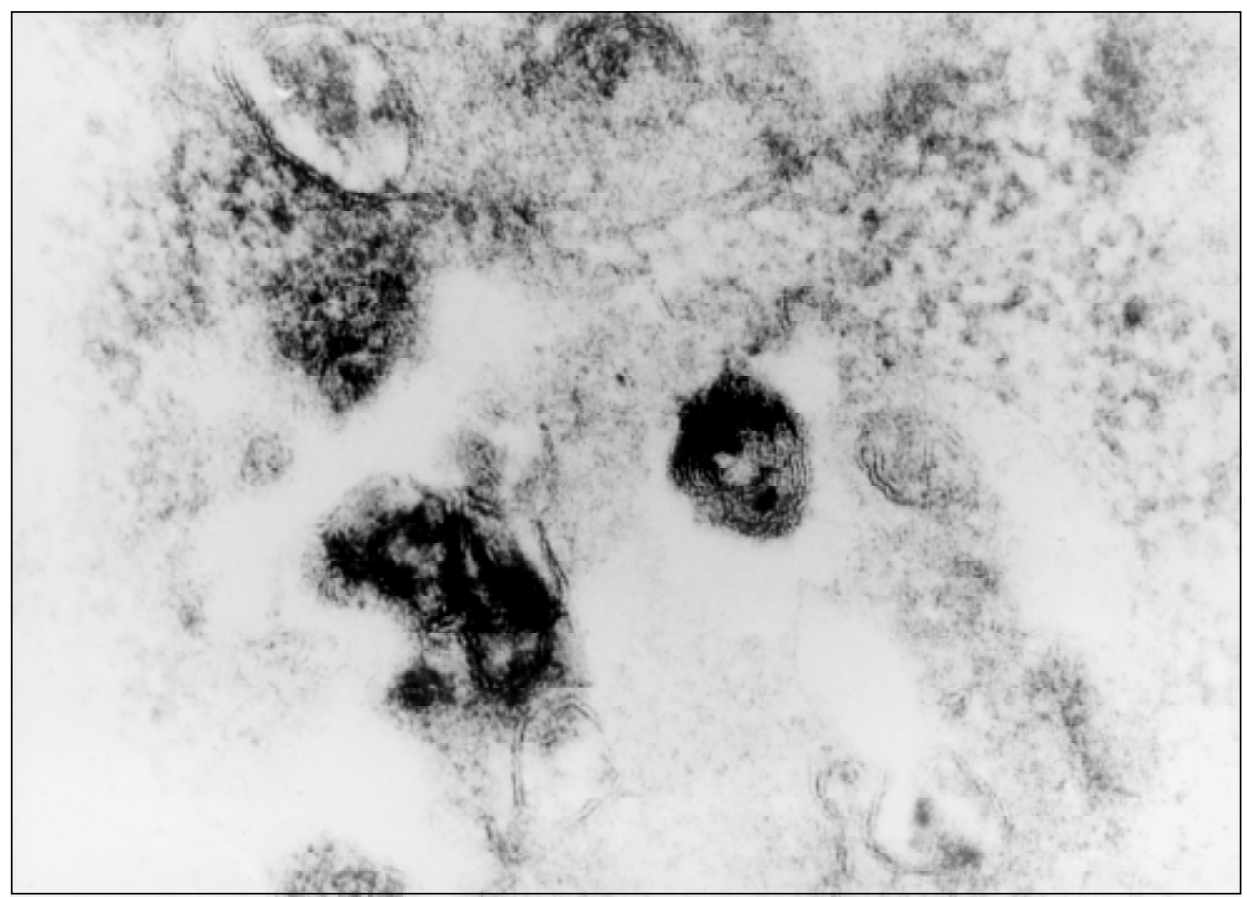

Fig 3. Fingerprint profile, $X, 85,000$.

Neurophysiological findings. EEGs revealed progressive disorganization of background activity with either focal paroxysms or diffuse bursts of slow waves and spikes. In none of the patients typical responses to slow intermitent photic stimulation were found. Visual-evoked potentials (VEP) were not available for any of the patients.

Neuroradiological findings. Neuroradiological studies revealed interesting findings in sibs 13 and 14 at ages 12 and 15 years, respectively. In case 13, CT scan showed hypodensity of the white matter mainly on temporo-occipital regions. Some calcifications were also recognized in the transitional area between gray and white matter on temporal lobes. Patient 14 had a similar pattern on CT scan, along with a more proeminent widening of the lateral ventricules. Patients 15, 16, and 17 exams showed mainly cerebral atrophy within 1-5 years of disease.

Morphological findings. All the patients had FP on the ultrastructural study of their skin biopsies. Two sibs, patients 15 and 16, also showed GRODs.

DNA samples available from some of the patients are now being analysed.

\section{DISCUSSION}

There are no epidemiological data on NCL prevalence in Brazil and this is, to our knowledge, the first report on a series of cases, so a more systematic recording needs to be done. In this retrospective review of 17 cases of childhood NCL, 7 were INCL; 5 were LINCL; and 5 were JNCL. These proportions differ from those described in other series and reviews on the subject, where juvenile cases are far more frequent than the other forms of neuronal ceroid lipofuscinosis. In fact, the juvenile form of NCL is the most prevalent one throughout the world; except for Finland, where the CLN1 (the etiologic locus of INCL) has a comparative higher incidence ${ }^{3,9,15}$. The large number of INCL cases found in our series prompts the question wether the ratio corresponds to the actual NCLs incidences in Southern Brazil. It most probably reflects some bias, due to the small 
number of the whole series, and due to family awareness ( 5 of the 7 cases were familial), seeking testing when first signs of the disease appear in a second baby. The other possibility is that JNCL cases might be underrepresented. This diagnosis may be less frequently considered for older children and adolescents presenting with ataxia, dementia or behavioral problems, the first signs in the majority of our cases.

The high frequency of positive results (17 diagnoses of NCLs among 25 possible cases ultrastructuraly investigated) reflects the restrictive inclusion criteria adopted. Skin biopsies were only performed with: a clinical picture suggestive of degenerative disease, and the exclusion of the main inborn errors of metabolism studied by biochemistry - lysosomal, peroxisomal, organic acids and amino acids disorders. Only after obtaining normal results, the ultrastructural investigation was performed.

When we look at the recurrence frequencies among our cases, 10 out of these 17 patients are index cases. There are 5 kindreds: sibs 3, 4 and 5; uncle and nephew (Patients 6 and 7); sibs 10 and 11; sibs 13 and 14; sibs 15, 16 and 17. Among the other cases, there was a positive familial history in at least one of them (Case 8). In a random sample of children with progressive neurodegenerative disorders, the frequency of positive ultrastructural diagnoses would probably be smaller, as seen worldwide.

Among the INCL cases, patient 6 and, in a way, patient 7 are atypical. Although presenting the pentad of elements a diagnosis of NCL, patient 6 lived longer than expected for an INCL case; and his morphological study showed not only the traditional GRODs, but also CBs. This mixed pattern of ultrastructural findings, although less frequent, has already been discribed. ${ }^{28}$ Family history shows a twin brother and a nephew with typical INCL presentation and clinical course, both dying around 3 years of age. Ultrastructural analysis of the nephew (patient 7), however, showed CB and FP, not the classical finding in INCL electron microscopy. These three affected boys were related by females, and even considering the small size of the family, the intriguing possibility of an X-linked variant of NCL comes to mind.

The classical finding of LINCL are the CBs, sometimes mixed with FPs. Among our 5 cases, CBs were present in all but patient 10, whose biopsy showed GRODs. In another series of cases, some of the patients classified by clinical course, neurophysiological studies and age of onset as CLN2 ( the main etiology of LINCL), also had GRODs on ultrastructural analysis ${ }^{33}$. Since LINCL is a more genetically heterogeneous group, compared to the other NCL types, it is possible that clinical studies lacking molecular data - like the present one - classify under the same entity, patients with very different genetic etiologies.

The ultrastructural findings of JNCL and protracted juvenile neuronal ceriod lipofuscinosis (PJNCL) are FPs, sometimes mixed with $\mathrm{CBs}^{5,34}$. Further research established a juvenile form of NCL with granular osmiophilic bodies. The early-juvenile form of NCL has both curvilinear bodies and fingerprint profiles as the classical JNCL (CLN3), and is not possible to differentiate these clinical forms from each other by electron microscopy of a skin biopsy ${ }^{35}$. The list of atypical forms and presentations is long and labyrinthic, especially within the spectrum of LINCL ${ }^{23}$. We cannot rule out that some of the cases considered by us as CLN3 on the basis of age, clinicopathological and neurophysiological as well as biopsy data, may correspond to one of the CLN2 variants. Only molecular investigation could correctly classify these patients. Unfortunately, most of the patients in this series have already died before proper DNA storage could be done. For those patients whose material is available, molecular studies are being performed. The emphasis on clinical aspects of NCL serves two purposes:

First, even with the perspective of a possible complicated and sometimes blurred clinicopathological classification for the NCLs, clinicians must keep in mind that classifying the NCL type is the second step in the process. The key issue is to consider the diagnosis at all. In that regard, the pentad of symptoms (complete or not): motor disturbances, especially ataxia, visual impairment, seizures, dementia and familial occurrence should serve as a heuristic approach to suspect childhood NCL and consider peripheric lymphocytes or skin biopsy studies 5 . 
Second, ultrastructural analysis continues to be a "gold standard" for NCL investigation and diagnosis, especially for the index case, and in developing countries, where molecular analysis may be difficult. Once the inclusion pattern is identified, molecular studies can warrant a clearer and more reliable classification. Testing sibs at risk, and prenatal diagnosis is then made faster by direct molecular analysis, providing better genetic counselling.

Acknowledgments - We thank Drs I V Schwartz, T Félix , and R Pires for referring patients.

\section{REFERENCES}

1. Goebel HH. Morphologic diagnosis in neuronal ceroid lipofuscinosis. Neuropediatrics 1997;28:67-69.

2. Mole SE, Gardiner RM, Goebel HH. Workshop on "The genetic and molecular basis of the neuronal ceroid lipofuscinoses". London, UK, November 13-16,1997. Eur J Paed Neurol 1998;2:2 (A1-6).

3. Goebel HH, Sharp JD. The neuronal ceroid-lipofuscinoses. recent advances. Brain Pathol 1998;8:151-162.

4. Santavuori P. Neuronal ceroid-lipofuscinosis in childhood. Brain Dev 1988;10:80-83.

5. Goebel HH. The neuronal ceroid-lipofuscinoses. Semin Ped Neurol 1996;3:270-278.

6. Claussen M, Heim P, Knispel J, Goebel HH, Kohlschutter A. Incidence of neuronal ceroid-lipofuscinoses in West Germany:variation of a method for studying autosomal recessive disorders. Am J Med Genet 1992;42:536-538.

7. MacLeod PM, Dolman CL, Change E, et al. The neuronal ceroid-lipofuscinoses in British Columbia: a clinical epidemiologic and ultrastructural study. Birth Defects 1976;12:289-296.

8. Taratuto AL, Saccoliti M, Sevlever G, et al. Childhood neuronal ceroid-lipofuscinoses in Argentina. Am J Med Genet 1995;57:144-149.

9. Cardona F, Rosati E. Neuronal ceroid-lipofuscinoses in Italy: an epidemiological study. Am J Med Genet 1995;57:142-143.

10. Berkovic SF, Carpenter S, Andermann F, et al. Kufs disease: a critical reappraisal. Brain 1988;111:27-62.

11. Goebel HH. The neuronal ceroid-lipofuscinoses. J Child Neurol 1995;10:424-437.

12. Martin JJ. Adult type of neuronal ceroid-lipofuscinosis.J Inher Metab Dis 1993;16:237-240

13. William RE, Verloes A, Munroe PB, et al. Genetic analysis of adult onset neuronal ceroid liposfuscinosis ( Kufs disease). Eur J Paed Neurol 1998;2:2 (A11).

14. Kohlschutter A, Gardiner RM, Goebel HH. Human forms of neuronal ceroid-lipofuscinosis (Batten disease): consensus on diagnostic criteria, Hamburg 1992. J Inher Metab Dis 1993;16:241-244.

15. Haltia M, Rapola J, Santavuori P. Infantile type so-called neuronal ceroid lipofuscinosis: histological and electron microscopic studies. Acta Neuropathol (Berl) 1973;26:157-170.

16. Hagberg B, Sourander P, Svennerholm L. Late infantile progressive encephalopathy with disturbed polyunsaturated fat metabolism. Acta Paediatr Scand 1968;57:495-499.

17. Carpenter S, Karpati G, Andermann F. A type of juvenile cerebromacular degeneration characterized by granular osmiophilic deposits. J Neurol Sci 1973;18:67-87.

18. Wisniewski KE, Kida E, Patxot OF, Connell F. Variability in the clinical and pathological findings in the neuronal ceroid lipofuscinoses: review of data and observations. Am J Med Genet 1992;42:525-532.

19. Santavuori PJ, Rapola J, Sainio K, Raitta C. A variant of Jansky-Bielschowsky disease. Neuropediatrics 1982;13:135-141.

20. Santavuori PJ, Rapola J, Nuutila A, et al. The spectrum of Jansky-Bielschowsky disease. Neuropediatrics 1991;22:92-96.

21. Lake BD, Cavanagh NP. Early-juvenile Batten's disease - a recognizable subgroup distinct from other forms of Batten's disease. Analysis of five patients. J Neurol Sci 1978;36:265-271.

22. Dyken P. Reconsideration of the classification of the neuronal ceroid-lipofuscinoses. Am J Med Genet [Suppl] 1988;5:69-84.

23. Dyken P, Wisniewski K. Classification of the neuronal ceroid-lipofuscinoses: an expantion of the atypical forms. Am J Med Genet 1995;57:150-154.

24. Hellsten E, Vesa J, Speer MC, et al. Refined assignment of the infantile neuronal ceroid lipofuscinosis (INCL, CLN1) locus at 1p32: incorporation of linkage disequilibrium in multipoint analysis. Genomics 1993;16:720-725.

25. Mitchson HM, Williams RE, McKay TR, et al. Refined genetic mapping of juvenile-onset neuronal ceroid-lipofuscinosis on chromosome 16. J Inher Metab Dis 1993;16:339-341.

26. Sharp JD, Wheeler RB, Savukoski M, et al. Exclusion mapping of classical late infantile neuronal ceroid lipofuscinosis (Jansky-Bielschowsky disease, CLN2) (Letter). Eur J Hum Genet 1995;3:326-328.

27. Mole SE. Recent advances in the molecular genetics of the neuronal ceroid lipofuscinoses. J Inher Metab Dis 1996;19:269-274.

28. Sharp JD, Wheeler RB, Lake BD, et al. Loci for classical and a variant late infantile neuronal ceroid lipofuscinosis map to chromosomes 11p15 and 15q21-23. Hum Mol Genet 1997;6:591-595.

29. Bennet MJ, Hofmann SL. The neuronal ceroid-lipofuscinoses (Batten disease): a new class of lysosomal storage diseases. J Inher Metab Dis 1999;22:535-544.

30. Sainio K. Neurophysiological findings in neuronal ceroid lipofuscinoses. Neuropediatrics 1997;28:70.

31. Lyon G, Adams RD, Kolodny EH (eds). Late infantile progressive genetic encephalopathies (metabolic encephalopathies of the second year of life). Neurology of hereditary metabolic diseases in children. 2Ed. New York: McGraw-Hill, 1996:146-150.

32. Lyon G, Adams RD, Kolodny EH (eds). Childhood and adolescent hereditary metabolic disorders. Neurology of hereditary metabolic diseases in children. 2Ed. New York: McGraw-Hill, 1996:241-243.

33. Narducci N, Verga ML, Binelli S, et al. Neuronal ceroid-lipofuscinosis: a clinical and morphological study of 19 patients. Am J Med Genet 1995;57:137-141.

34. Goebel HH. Protracted juvenile neuronal ceroid-lipofuscinosis. J Inher Met Dis 1993;16:233-236.

35. Lake BD. The importance of adequate light and electron microscopical observations in defining the type of NCL. Eur J Paed Neurol 1998;2:2 (A14). 